\title{
Recubrimiento por láser de aleaciones cuasicristalinas
}

\author{
F. Audebert ${ }^{(*)}$, R. Colaço ${ }^{(* *)}$, R. $\operatorname{Vilar}^{(* *)}$ y H. $\operatorname{Sirkin}^{(*)}$
}

\begin{abstract}
Resumen Los cuasicristales constituyen un nuevo tipo de estructuras ordenadas con características metaestables a temperatura ambiente. En general, en determinadas aleaciones, pueden obtenerse por enfriamiento rápido desde el estado líquido. Han demostrado poseer buenas propiedades para su aplicación como recubrimientos resistentes al desgaste y a la corrosión. En este sentido, desde hace unos años, se ha impulsado el desarrollo de su aplicación mediante técnicas de thermal spray, aunque no se han encontrado referencias de su aplicación mediante técnicas de láser. En este trabajo, se presenta la formación de cuatro recubrimientos de aleaciones con composiciones cuasicristalinas sobre un sustrato de aluminio, utilizando un láser continuo de $\mathrm{CO}_{2}$, con inyección simultánea del material en forma de mezcla de polvos. Los mismos fueron caracterizados por difracción de rayos X, microscopía electrónica de barrido y microdureza Vickers.
\end{abstract}

Palabras clave: Aleaciones de aluminio. Cuasicristales. Recubrimientos por láser.

\section{Laser cladding of quasicrystalline alloys}

\begin{abstract}
Quasicrystals are a new class of ordenated structures with metaestable characteristics at room temperature. Quasicrystalline phases can be obtained by rapid quenching from the melt of some alloys. In general, quasicrystals present properties which make these alloys promising for wear and corrosion resistant coatings applications. During the last years, the development of quasicrystalline coatings by means of thermal spray techniques has been impulsed. However, no references have been found of their application by means of laser techniques. In this work four claddings of quasicrystalline compositions formed over aluminum substrate, produced by a continuos $\mathrm{CO}_{2}$ laser using simultaneous powders mixture injection are presented. The claddings were characterized by $\mathrm{X}$ ray diffraction, scanning electron microscopy and Vickers microhardness.
\end{abstract}

Keywords: Aluminum alloys. Quasicrystals. Laser claddings.

\section{INTRODUCCIÓN}

Los cuasicristales son una nueva forma de la materia condensada. Su estructura, a escala atómica de largo alcance, no es desordenada, como la de los vidrios o la de los líquidos, ni tampoco está compuesta por una translación de celdas unitarias. $\mathrm{Su}$ estructura no puede explicarse por las reglas de la cristalografía clásica. Son sólidos con un ordenamiento de tipo cuasiperiódico. Desde su descubrimiento por Shechtman et al. (1) en 1984, en una aleación de Al-Mn obtenida por solidificación rápida, se han encontrado cuasicristales con diferentes

(*) Lab. de Sólidos Amorfos. Fac. de Ingeniería. Univ. de Buenos Aires. Po. Colón 850. Ciudad de Buenos Aires (1063) (Argentina).

(**) Dpto. de Engenharia de Materiais. Instituto Superior Técnico de Lisboa. Av. Rovisco Pais (1096) Lisboa (Portugal). simetrías de rotación y en diferentes tipos de sistemas (2). Los más comunes son los llamados "icosaédricos" (1), que poseen seis ejes de simetría de orden cinco. Algunos de ellos, como en el sistema Al$\mathrm{Cu}-\mathrm{Fe}$, se han manifestado como fases estables a alta temperatura (3).

Estas estructuras cuasiperiódicas, en las aleaciones de base aluminio, presentan, a temperatura ambiente, módulos de Young, $E$, entre un 50 y un 100 $\%$ más elevados que el del aluminio (4), altos valores de dureza, aunque muy baja tenacidad, y muy bajos coeficientes de fricción. Han demostrado ser resistentes a la corrosión y a la oxidación (5) y presentan muy bajas conductividades, tanto térmica como eléctrica, respecto a los materiales metálicos convencionales, siendo similares a las de los óxidos aislantes (6).

La fabricación en volumen de los cuasicristales es extremadamente dificultosa, debido a caracterís- 
ticas inherentes a la particular simetría de su estructura y al requerimiento de condiciones de enfriamiento rápido. Esta dificultad de producción en volumen y su alta fragilidad a temperatura ambiente han conducido los esfuerzos relativos a su aplicación hacia la forma de recubrimientos protectores (5 y 7). En este sentido existen patentes de aplicación de cuasicristales por técnicas de thermal spray (8). Las propiedades tribológicas sobre estos recubrimientos han sido estudiadas (9), encontrándose altos valores de microdureza $(\mu \mathrm{Hv}=5-6 \mathrm{GPa})$ y muy bajos coeficientes de fricción $(\mu \approx 0,2)$.

$\mathrm{El}$ procesamiento de materiales por láser es una técnica en expansión que permite el tratamiento de superficies bajo dos formas bien diferenciadas: sin fusión superficial y con fusión superficial. Dentro de estas últimas aplicaciones, se encuentra la conocida como laser cladding, que consiste en la generación de un recubrimiento de un material diferente al sustrato, en general, con inyección simultánea de polvos durante la fusión superficial por el haz de láser. Su primera referencia de aplicación data de 1981 (10) y numerosos trabajos han demostrado la fiabilidad de su aplicación, que va desde aleaciones metálicas (11 y 12) hasta cerámicos (13). Este tratamiento permite obtener recubrimientos de gran homogeneidad y refinamiento estructural, siendo posible controlar la forma de la pista y el grado de dilución del material aplicado con el sustrato, lo cual proporciona una mejor adherencia que las técnicas de thermal spray.

\section{PARTE EXPERIMENTAL}

Se han seleccionado cuatro composiciones de aleaciones formadoras de cuasicristales por enfriamiento rápido bien estudiadas en la literatura (Tabla I). Algunas de ellas ya ensayadas por deposición desde técnicas de thermal spray.

La preparación de las muestras de recubrimiento por láser se realizó sobre un sustrato de aluminio, con inyección simultánea de mezclas de polvos con las composiciones seleccionadas. Al sustrato de aluminio, de pureza comercial, se le aplicó un tratamiento de arenado previo a la aplicación del recubrimiento por láser.
TABLA II.-Características de los polvos utilizados: pureza y tamaño de partícula.

TABLE II.- Powders characteristics: purity and particle size.

\begin{tabular}{|c|c|c|c|c|}
\hline Elemento & $\mathrm{Al}$ & $\mathrm{Cu}$ & $\mathrm{Fe}$ & $\mathrm{Cr}$ \\
\hline Pureza $(\%)$ & 99.5 & 99.5 & 99.5 & $99 \mathrm{~min}$. \\
\hline Tamaño $(\mu \mathrm{m})$ & 150 & 25 & 75 & 75 \\
\hline
\end{tabular}

Para la mezcla de polvos se utilizaron polvos de elementos de pureza comercial. Las características de los mismos se ofrecen en la tabla II.

Para reducir la humedad adsorbida, las mezclas de polvos se secaron a $90{ }^{\circ} \mathrm{C}$ durante $2 \mathrm{~h}$ antes de ser inyectadas.

Se utilizó un láser continuo de $\mathrm{CO}_{2}$ de $3 \mathrm{~kW}$ de potencia máxima. Para reducir la oxidación del metal fundido, se aplicó gas argón sobre la superficie de ląs muestras durante cada etapa del procesamiento por láser.

La aplicación del recubrimiento (clad) se realizó en tres etapas bien definidas. La primera etapa consistió en la real aplicación del recubrimiento mediante la eyección y fusión de las mezclas de polvos. Esta etapa se efectuó en dos pasos: un primer depósito de material con fusión parcial del sustrato y un segundo depósito de la mezcla de polvos para obtener un mayor sesgo en la composición química del recubrimiento, dado que el primer depósito se pudo ver afectado en su composición química por dilución del sustrato. En esta etapa, se empleó una densidad de potencia de $1,6 \times 10^{5} \mathrm{~W} / \mathrm{cm}^{2}$ con un solape del $20 \%$ entre cada cordón. La velocidad de barrido (entre 4 y $8 \mathrm{~mm} / \mathrm{s}$ ) y el caudal de polvos inyectado (entre 0,025 y $0,085 \mathrm{~g} / \mathrm{s}$ ) fueron combinados para cada aleación y cada paso, de forma tal que se obtuviese un recubrimiento final con un espesor alrededor de $1 \mathrm{~mm}$.

La segunda etapa consistió en la refusión del recubrimiento aplicado con objeto de eliminar defectos (físicos y químicos) y homogeneizar y reafinar la estructura en las capas superficiales del recubrimiento. La densidad de potencia utilizada fue de

TABLA I.-Composición química de las mezclas de polvos utilizadas y su nomenclatura

TABLE I.-Chemical composition of the mixed powders and designation.

\begin{tabular}{|c|c|c|c|c|}
\hline $\mathrm{N}^{\mathrm{o}}$ & QC-1 & QC-2 & QC-3 & QC-4 \\
\hline Comp. Química (\%at) & $\mathrm{Al}_{63} \mathrm{Cu}_{25} \mathrm{Fe}_{12}$ & $\mathrm{Al}_{65} \mathrm{Cu}_{19} \mathrm{Fe}_{8} \mathrm{Cr}_{8}$ & $\mathrm{Al}_{76} \mathrm{Fe}_{12} \mathrm{Cr}_{12}$ & $\mathrm{Al}_{86} \mathrm{Cr}_{14}$ \\
\hline Referencias & $(3,5)$ & $(7)$ & $(14)$ & $(15)$ \\
\hline
\end{tabular}


$1,9 \times 10^{5} \mathrm{~W} / \mathrm{cm}^{2}$, con una velocidad de barrido de 5 $\mathrm{mm} / \mathrm{s}$ y un solape del $40 \%$ entre cada pasada.

La tercera etapa consistió en una segunda refusión con una densidad de potencia de $3,18 \times 10^{5}$ $\mathrm{W} / \mathrm{cm}^{2}$ y una velocidad de barrido de $1.150 \mathrm{~mm} / \mathrm{s}$. Esta segunda refusión a alta velocidad es la responsable de generar las condiciones de enfriamiento rápido para dar lugar a la formación de estructuras cuasicristalinas.

Las pasadas de láser en las etapas de refusión se aplicaron en forma transversal a las etapas anteriores y, después de cada etapa, se lijó la superficie.

La composición química superficial de cada muestra se verificó mediante análisis de rayos $\mathrm{X}$ por dispersión de energía (EDAX). La estructura del recubrimiento se observó por microscopía electrónica de barrido (MEB) y difracción de rayos $\mathrm{X}$ de incidencia rasante (DRX-IR), con un ángulo $\theta=$ $8^{\circ}$ y radiación de $\mathrm{Cu}-\mathrm{K} \alpha$. Las propiedades mecánicas del recubrimiento se determinaron mediante ensayos de microdureza Vickers con carga de $200 \mathrm{~g}$.

\section{RESULTADOS Y DISCUSIÓN}

En la tabla III se muestra el promedio de cinco determinaciones mediante EDAX de la composición química superficial de cada muestra y los valores medios con su desviación estándar de 10 mediciones de la microdureza en la superficie de los recubrimientos.

Los resultados del análisis de la composición química final en la superficie de los recubrimientos indican que todas las muestras han sufrido alguna variación composicional respecto de la concentración nominal de las mezclas de polvos inyectadas. Estas desviaciones no son cuantitativamente importantes, salvo en la muestra QC-2, cuya composición nominal fue formulada a partir de la QC-1 por agregado de cromo, obteniéndose finalmente una composición química similar a la aleación QC-3 aleada con cobre. La variación composicional producida en este caso, posiblemente se deba a una deficiencia en el mezclado de los polvos componentes. No obstante según lo observado en los difractogramas, estas variaciones en lo que se refiere a la composición no parecen afectar en gran medida la formación de la fase cuasicristalina.

Los valores de la microdureza son superiores a los que presentan las clásicas aleaciones endurecidas de aluminio y concuerdan con los que se reseñan en la literatura para aleaciones cuasicristalinas (16). Las aleaciones que contienen cobre son las que presentan los valores más altos, mientras que la muestra de composición QC-4, que es la que tiene menor proporción de fase icosaédrica, es la que presenta los valores de microdureza más bajos.

Por otra parte, a excepción de la muestra QC-4, las otras aleaciones mostraron distinto grado de fisuración durante el enfriamiento, siendo este más importante en la muestra QC-1.

La observación mediante MEB de secciones transversales a las pistas reveló que, después de la refusión a baja velocidad (segunda etapa), todos los recubrimientos presentan una estructura celular dendrítica homogénea y exenta de porosidad. La refusión a alta velocidad (tercera etapa) originó una capa superficial con un espesor promedio de $50 \mu \mathrm{m}$, muy homogénea y de estructura extremadamente fina que, a modo de ejemplo, en las figuras 1 y 2 se puede observar la homogeneidad y el refinamiento estructural logrado en la capa superior del recubri-

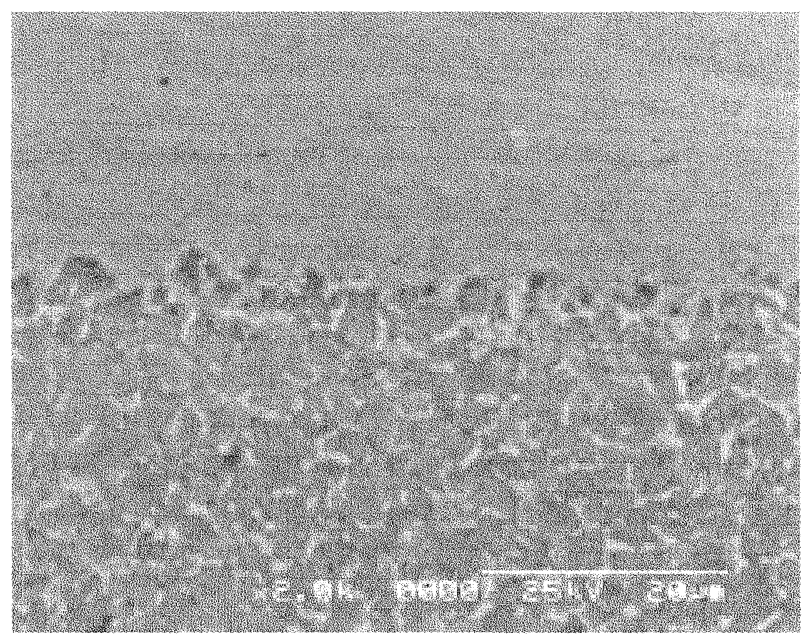

FIG. 1.- Imagen SEM de la probeta QC-2.

FIG. 1.-SEM image of $Q C$-2 sample.

TABLA III.- Composición química resultante y microdureza Vickers determinadas sobre la superficie de cada recubrimiento.

TABLE III.-Resulting chemical composition and Vickers hardness measured on the surface of each cladding.

\begin{tabular}{|c|c|c|c|c|}
\hline $\mathrm{N}^{\mathrm{o}}$ & $\mathrm{QC}-1$ & $\mathrm{QC}-2$ & $\mathrm{QC}-3$ & $\mathrm{QC}-4$ \\
\hline C. Q. (\%at) & $\mathrm{Al}_{64.5} \mathrm{Cu}_{24.5} \mathrm{Fe}_{11}$ & $\mathrm{Al}_{77} \mathrm{Cu}_{6} \mathrm{Fe}_{8} \mathrm{Cr}_{9}$ & $\mathrm{Al}_{77.5} \mathrm{Fe}_{12} \mathrm{Cr}_{10.5}$ & $\mathrm{Al}_{84} \mathrm{Cr}_{16}$ \\
\hline $\mathrm{Hv}_{0.2}$ & $725 \pm 50$ & $720 \pm 20$ & $655 \pm 50$ & $465 \pm 50$ \\
\hline
\end{tabular}




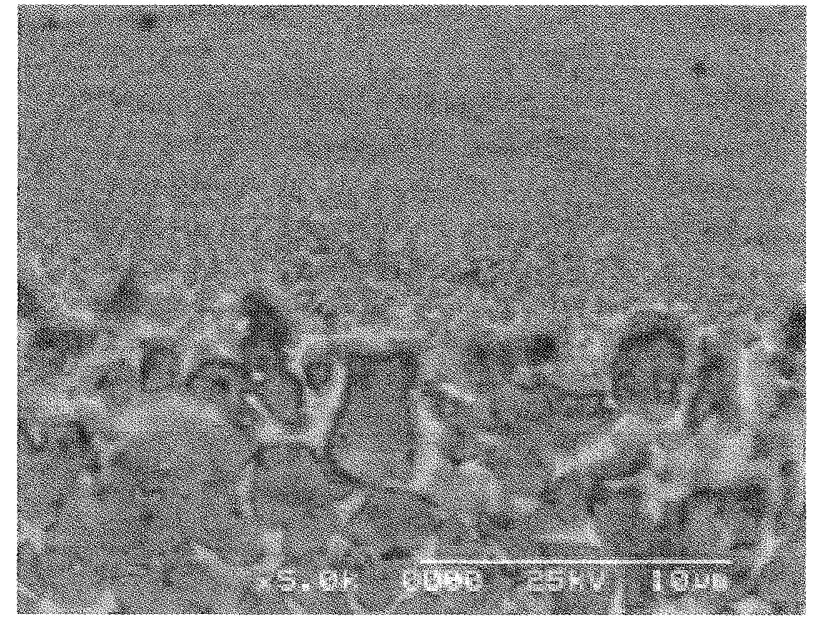

FIG. 2.- Imagen SEM de la probeta QC-2.

FIG. 2._- SEM image of QC-2 sample.

miento QC-2, como efecto de la refusión a alta velocidad (tercera etapa), respecto de la estructura obtenida después de la segunda etapa (parte inferior de las figuras 1 y 2 ).

La figura 3 muestra los difractogramas de rayos $\mathrm{X}$ con la correspondiente identificación de fases. Todas las muestras presentan fase icosaédrica en distintas proporciones

La muestra QC-1 presenta, además de la fase cuasicristalina, la fase cúbica AlFe. Las mismas fases, y en proporciones similares, fueron obtenidas en recubrimientos producidos por thermal spray (5).

La muestra QC-2 está prácticamente compuesta sólo por fase $i$, lo cual concuerda con los resultados obtenidos por Dubois (7) mediante thermal spray. La deficiencia en cobre, respecto de la composición nominal, y de la utilizada en (7), sólo produce un corrimiento de las líneas de difracción en dirección hacia un aumento del "parámetro del cuasicristal" (17).

El difractograma de la aleación QC-3, indica la presencia de la fase $\mathrm{Al}_{13} \mathrm{Fe}_{4}$, además de la fase $i$.

El recubrimiento QC-4 es el que presentan la menor proporción de fase $i$. Está formado principalmente por las fase $\mathrm{Al}_{13} \mathrm{Cr}_{2}$ y una pequeña proporción de $\mathrm{Al} \alpha$.

Las principales líneas de las fases $\mathrm{Al}_{13} \mathrm{Fe}_{4}$, $\mathrm{Al}_{13} \mathrm{Cr}_{2}$ y de la fase icosaédrica se encuentran concentradas entre 40 y $45^{\circ}$ en $2 \theta_{(\mathrm{Cu}-\mathrm{K} \alpha)}$ y son de difícil separación, no obstante la línea $(-111)$ en $2 \theta_{(\mathrm{Cu}-\mathrm{K} \alpha)}$ $=14,6^{\circ}$ y la $(101000)$ alrededor de $2 \theta_{(\mathrm{Cu}-\mathrm{K} \alpha)}=75^{\circ}$, son las líneas más características en la identificación de la fases $\mathrm{Al}_{13} \mathrm{Cr}_{2}$ y de la fase cuasicristalina, respectivamente (18 y 15$)$.

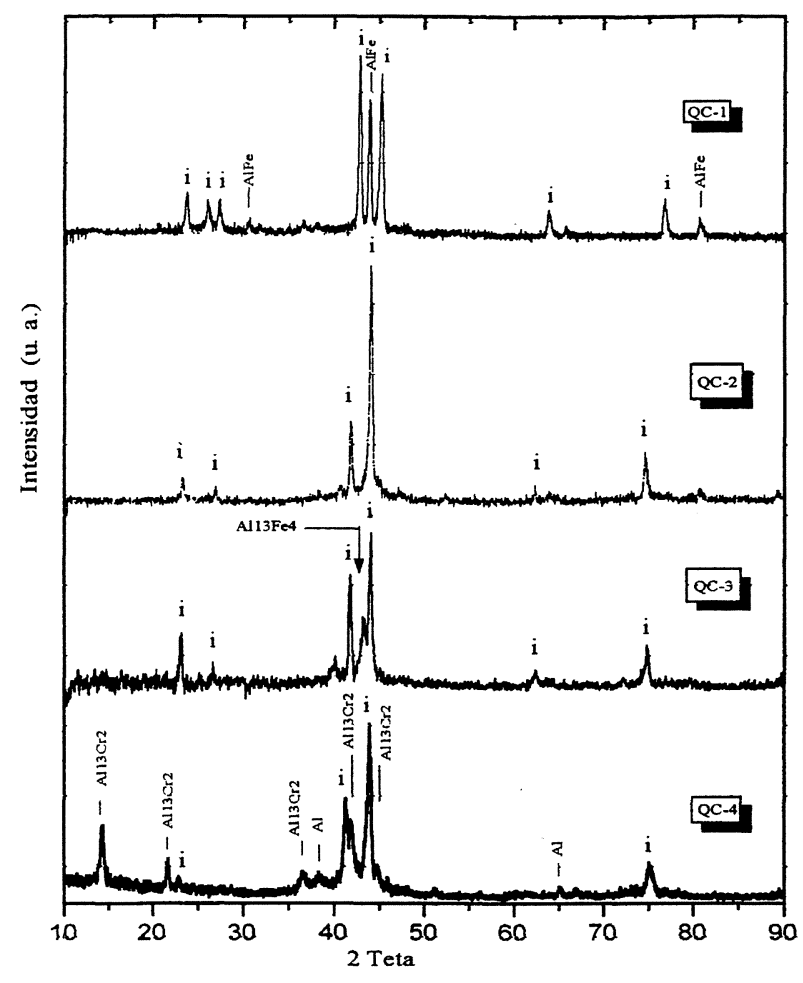

FIG. 3.- Difractogramas de rayos $\mathrm{X}$ con incidencia rasante. i: Fase icosaédrica.

FIG. 3.- Low angle X-Ray Spectra. o: Icosaedric phase.

\section{CONCLUSIONES}

Se han producido recubrimientos mayoritariamente cuasicristalinos mediante la técnica láser con inyección simultánea de polvos premezclados. De lo cual no se han encontrado referencias en la literatura.

El proceso final, de refusión a alta velocidad, produce en estas aleaciones una capa superficial de alrededor de $50 \mu \mathrm{m}$ con una estructura extremadamente fina, homogénea y exenta de porosidad.

Todos los recubrimientos presentaron altos valores de microdureza, siendo las muestras con cobre las que mostraron los valores más elevados, alrededor de $\mathrm{Hv}_{0,2}=720$.

\section{Agradecimiento}

Este trabajo fue realizado como producto de las colaboraciones surgidas en el marco del Subprograma "Procesamiento de Materiales por Láser" de la Red CYTED. 


\section{REFERENCIAS}

(1) Shechtman D., Blech, I., Gratias y D. Cahn, J. Phys. Rev. Lett. 53, 1984: 1951.

(2) TsAI, A.P. MRS Bull. 22 (11), 1997: 43.

(3) Gayle, F., Shapiro, A., Biancniello, F. y Boettinger, W. Metall. Trans. 23 A, 1992: 2.409.

(4) Dubois, J.M. in Trends in Non-Crystalline Solids, Proc. 3rd Intern. Worshop on Non-Cryst. Solids. Eds. A. Conde, C.F. Conde, C.F. y Millán, M. Word Scientific. 1991: 343.

(5) Besser, M.F. y Eisenhammer, T. MRS Bull. 22 (11), 1997: 59.

(6) Archambault, P. y Janot, C., MRS Bull. 22 (11), 1997: 48.

(7) Dubois, J.M, Kang, S.S. y Perrot, A. Mat. Sci. \& Eng. A-179/A-180 (1994: 122.

(8) Dubois, J.M. y Weinland, P. Pat. Francesa núm. 2.635.117 (30 de abril de 1993).
(9) Kang, S.S., Dubois, J.M. y Stebut, J. J. Mater. Res. 8 (10), 1993: 2.471.

(10) Macintyre, R.M. The use of lasers in Rolls Royce. Eds. C.W. Draper y P. Mazzoldi. Laser Surface Treatment of Metals. 1986: 545.

(11) Carvalho, D., Cardoso, S. y Vilar R. Scripta Mat. 37 (4), 1997: 523

(12) Tiziani, A., Giordano, L., Matteazzi, P. y Badan, B. Mat. Sci. \& Eng. 88, 1987: 171.

(13) Ramouse, E., Giordano, L., Tiziani, A., Badan, B. y CANTEllo, M. Key Eng. Mat. 47, 1990: 425.

(14) Ziani, M.G., Pianelli, A., Redjaimia, A., Zahra, C.Y. y ZAHRA A.M. J. Mat. Sci. 30, 1995: 2.921.

(15) Inoue, A., Kimura, H. y Masumoto, T. J. Mat. Sci. 22,1987: 1.758 .

(16) Kang, S.S. y Dubois, J.M. Phil. Mag. A, 66 (1), 1992: 151.

(17) Manaila, R., Florescu, V., Jianu, A. y Radulescu, O. Phil. Mag B. 60 (5), 1989: 589.

(18) DaSilva, R.C., Sousa, R.C., Conde, O., DaSilva, M.F. y SoARES, J.C. Surf. \& Coat. Tech. 83, 1996: 60. 